AN. MED. INTERNA (Madrid) Vol. 18, N. ${ }^{\circ}$, pp. 309-311, 2001

\title{
Diferencias entre la espondilitis tuberculosa y la espondilitis brucelar
}

\author{
J. M. CALVO ROMERO, J. L. RAMOS SALADO, F. GARCÍA DE LA LLANA*, \\ J. C. BUREO DACAL, P. BUREO DACAL, M. PÉREZ MIRANDA \\ Servicio de Medicina Interna. *Unidad de Enfermedades Infecciosas. Hospital Regional \\ Universitario Infanta Cristina. Badajoz
}

\author{
DIFFERENCES BETWEEN TUBERCULOUS SPONDYLITIS AND \\ BRUCELLAR SPONDYLITIS
}

\section{RESUMEN}

Objetivo: Identificar posibles diferencias en las características clínicas y analíticas entre la espondilits tuberculosa (ET) y la espondilitis brucelar (EB).

Pacientes y métodos: Estudio retrospectivo de los pacientes diagnosticados de ET y EB en nuestro hospital entre enero de 1992 y diciembre de 1998.

Resultados: Diecisiete pacientes fueron diagnosticados de ET y 10 de EB. La ET se caracterizó en nuestra serie por un mayor tiempo de evolución de la clínica hasta el momento del diagnóstico $(27,9 \pm 24,6 v s$. $16 \pm 5,6$ semanas, $p=0,02$ ). Encontramos una mayor frecuencia, pero sin alcanzar la significación estadística, de inmunosupresión, de uno o varios abscesos paravertebrales y/o epidurales, de compresión medular, de anemia y de velocidad de sedimentación globular elevada en la ET, y una mayor frecuencia de fiebre/febrícula y de dolor vertebral residual en la EB. La localización lumbar fue la más frecuente en ambos grupos (58,8\% en la ET y $70 \%$ en la EB).

Conclusiones: Es posible que existan algunas diferencias en las características clínicas y analíticas de la ET y de la EB que puedan ser de ayuda en el diagnóstico diferencial de ambas entidades y orientar el tratamiento empírico en los casos sin un diagnóstico definitivo microbiológico o a la espera de la confirmación diagnóstica.

PALABRAS CLAVE: Espondilitis. Espondilitis tuberculosa. Espondilitis brucelar.

\begin{abstract}
Objective: To identify potential differences in the clinical and labo ratory characteristics between tuberculous spondylitis (TS) and bruce llar spondylitis $(B S)$.

Patients and methods: Retrospective study of patients with TS and BS diagnosed in our hospital between january 1992 and december 1998.

Results: TS was diagnosed in 17 patients and BS in 10 patients. In our series, a higher delay in the diagnosis $(27.9 \pm 24.6$ vs. $16 \pm 5.6$ weeks, $p=0,02$ ) was found in TS. There was a higher frequency, but wit hout stadistic significance, of immunosupression, one or several para vertebral or epidural abscesses, spinal cord compression, anemia and an elevated erythrocyte sedimentation rate in TS, and a higher frequency of fever/febricule and residual vertebral pain in BS. Lumbar location was the most frequent in both groups $(58.8 \%$ in TS and $70 \%$ in BS).

Conclusions: It is possible that there were some differences in the clinical and laboratory characteristics between TS and BS which may be an aid in the differential diagnosis of both entities and orient the empiri cal treatment in these cases without a definitive microbiological diagno sis or while awaiting the diagnostic confirmation.
\end{abstract}

KEY WORDS: Spondylitis. Tuberculous spondylitis. Brucellar spondyli tis.

Calvo Romero JM, Ramos Salado JL, García de la Llana F, Bureo Dacal JC, Bureo Dacal P, Pérez Miranda M. Diferencias entre la espondi litis tuberculosa y la espondilitis brucelar. An Med Interna (Madrid) 2001; 18: 309-311.

\section{INTRODUCCIÓN}

Un $1-5 \%$ del total de casos de tuberculosis (TBC) y un 5$15 \%$ de los casos de TBC extrapulmonar corresponden a formas osteoarticulares (1-3). La espondilitis tuberculosa (ET) es la forma más frecuente de TBC osteoarticular (2,3). La ET supone un $20-25 \%$ del total de las espondilitis infecciosas en nuestro medio $(4,5)$. La espondilitis brucelar (EB) se presenta en un 6-12\% de los casos de brucelosis (6-8), y constituye un $20-50 \%$ de las espondilitis infecciosas en nuestro medio $(4,5)$. Con frecuencia en la ET no existen síntomas ni signos de afectación pulmonar ni sistémica (2). Además las pruebas de imagen (radiología simple, tomografía computadorizada (TC), resonancia magnética y gammagrafía) tienen escaso valor en el diagnóstico diferencial entre la ET y la EB $(9,10)$.
La realización de una prueba diagnóstica invasiva (biopsia abierta, biopsia percutánea con control de TC o punción-aspiración con control de $\mathrm{TC}$ ) es necesaria para conseguir una confirmación microbiológica.

En el presente estudio comparamos, con la intención de encontrar posibles diferencias que puedan ser de ayuda en el diagnóstico diferencial, las características clínicas y analíticas de nuestra serie de pacientes con ET y EB, así como los resultados obtenidos tras completar el tratamiento médico o médico-quirúrgico.

\section{PACIENTES Y MÉTODOS}

Realizamos un estudio retrospectivo de los pacientes diagnosticados de ET o EB entre enero de 1992 y diciembre de 1998 en el

Trabajo aceptado: 1 de Febrero de 2001

Correspondencia: José María Calvo Romero. Héroes de Cascorro, 9, $3^{\circ}$ A. 06004 Badajoz. 
Hospital Infanta Cristina de Badajoz, hospital regional de la Comunidad Autónoma de Extremadura (cuyos servicios de Traumatología y de Neurocirugía son de referencia en cirugía de la columna vertebral para toda la comunidad autónoma). El diagnóstico de ET se estableció por: a) el aislamiento de Mycobacterium tuberculosis en el cultivo en medio de Löwenstein-Jensen de las muestras vertebrales o paravertebrales obtenidas mediante biopsia y/o punciónaspiración, o b) la presencia de un cuadro clínico-radiológico compatible con Mantoux positivo, acompañado de respuesta al tratamiento anti-tuberculoso. El diagnóstico de EB se estableció por: a) el aislamiento de Brucella en las muestras vertebrales o paravertebrales obtenidas mediante biopsia y/o punción aspiración, o b) la existencia de un cuadro clínico-radiológico y serología compatibles (seroaglutinación de Wright $>1 / 160$, inmunofluorescencia indirecta $>1 / 100$, prueba de Coombs anti-Brucella $>1 / 320$ ), acompañado de respuesta al tratamiento anti-Brucella.

Se recogieron las características clínicas y analíticas en el momento del diagnóstico, así como el resultado final tras completar tratamiento médico o médico-quirúrgico. Las características numéricas se expresaron como media \pm desviación standard. El análisis estadístico se realizó mediante el programa EPI-INFO 5.01 utilizando la prueba $\chi^{2}$ corregida por Yates o el test exacto de Fisher, cuando alguno de los valores esperados fue menor de 5, para la comparación de proporciones y la prueba de la " $t$ " de Student para la comparación de medias. Se consideró estadísticamente significativo un valor de p inferior a 0,05 .

\section{RESULTADOS}

Identificamos 17 pacientes con el diagnóstico de ET y 10 pacientes con el diagnóstico de EB. Se realizó biopsia y/o punción-aspiración de las lesiones vertebrales o paravertebrales en 11 de los casos $(64,7 \%)$ de ET, aislándose Mycobacterium tuberculosis en 10 casos. En los otros 7 casos de ET el diagnóstico se estableció por la presencia de un cuadro clínicoradiológico compatible con Mantoux positivo, acompañado de respuesta al tratamiento anti-tuberculoso. En 6 pacientes (60\%) con EB se realizó biopsia y/o punción-aspiración, resultando el cultivo de las muestras positivo para Brucella en 4 casos. En los otros 6 casos de EB el diagnóstico se estableció por la existencia de un cuadro clínico-radiológico-serológico compatible acompañado de respuesta al tratamiento anti-Bru cella. La rentabilidad diagnóstica de la biopsia y/o punciónaspiración de las lesiones vertebrales o paravertebrales fue del $90,9 \%$ y del $66,6 \%$ para la ET y la EB, respectivamente.

Veinte casos del total $(74,1 \%)$ fueron varones. La edad media de los pacientes fue 54,9 años (rango 16-83 años). Dieciocho pacientes $(66,6 \%)$ tuvieron una edad igual o superior a 50 años en el momento del diagnóstico. El tiempo medio de evolución de la clínica hasta el momento del diagnóstico fue 23,9 semanas (intervalo 3-70 semanas). Sólo 5 casos (18,5\%) presentaron una causa de inmunosupresión conocida: infección por el virus de la inmunodeficiencia humana (VIH) en 2 casos, diabetes melitus tipo 2 en 2 casos y cirrosis hepática en 1 caso.

La localización más frecuente en la ET fue la lumbar $(58,8 \%)$, al igual que en la EB $(70 \%)$. Sólo en 1 caso de ET y en otro caso de EB hubo afectación de más de 2 cuerpos vertebrales. Un $35,2 \%$ de los casos de ET y un $20 \%$ de los casos de EB presentaron compresión medular, y todos ellos se sometieron a cirugía descompresiva. En las tablas I y II se comparan las características clínicas y analíticas de los grupos de ET y EB.
TABLA I

CARACTERÍSTICAS CLÍNICAS: CO M PARACIÓ N ENTRE ESPO NDILITS TUBERCULOSA (ET) Y ESPO NDILITIS BRUCELAR (EB)

\begin{tabular}{lccc}
\hline & $E T(n=17)$ & EB $(n=10)$ & $p$ \\
\hline Edad (años) & $58,1 \pm 15,1$ & $49,7 \pm 19,1$ & NS \\
Edad $\geq 50$ años & $70,5 \%$ & $60 \%$ & NS \\
Sexo (varón) & $76,4 \%$ & $70 \%$ & NS \\
Inmunosupresión & $30,7 \%$ & $10 \%$ & NS \\
Infección VIH & $13,3 \%$ & $0 \%$ & NS \\
Tiempo de evolución (semanas) & $27,9 \pm 24,6$ & $16 \pm 5,6$ & 0,02 \\
Fiebre/ febrícula & $23,5 \%$ & $50 \%$ & NS \\
Dolor vertebral & $88,2 \%$ & $100 \%$ & NS \\
Pérdida de peso & $23,5 \%$ & $30 \%$ & NS \\
Localización cervical & $0 \%$ & $10 \%$ & NS \\
Localización dorsal & $47 \%$ & $20 \%$ & NS \\
Localización lumbar & $58,8 \%$ & $70 \%$ & NS \\
Localización sacra & $5,8 \%$ & $10 \%$ & NS \\
Absceso/s paravertebrales & & & \\
y/o epidurales & $70,5 \%$ & $50 \%$ & NS \\
Discitis & $100 \%$ & $100 \%$ & NS \\
Afectación de más de 2 vértebras & $5,8 \%$ & $10 \%$ & NS \\
Compresión medular & $35,2 \%$ & $20 \%$ & NS \\
Secuelas tras tratamiento & $35,2 \%$ & $60 \%$ & NS \\
Dolor vertebral residual & $35,2 \%$ & $60 \%$ & NS \\
Déficit motor residual & $11,7 \%$ & $10 \%$ & NS
\end{tabular}

$\mathrm{VIH}$ : virus de la inmunodeficiencia humana. NS: no significación estadística $(p \geq 0,05)$.

TABLA II

CARACTERÍSTICAS ANALÍTICAS: COM PARACIÓ N ENTRE ESPO NDILITS TUBERCULOSA (ET) Y ESPO NDILITIS BRUCELAR (EB)

\begin{tabular}{lccc}
\hline & $E T(n=17)$ & $E B(n=10)$ & $p$ \\
\hline Hemoglobina $(\mathrm{g} / \mathrm{dl})$ & $12,6 \pm 0,9$ & $13 \pm 1,4$ & $\mathrm{NS}$ \\
Hematocrito $(\%)$ & $37,4 \pm 3,1$ & $37,2 \pm 3,8$ & $\mathrm{NS}$ \\
Anemia (hemoglobina $<12 \mathrm{~g} / \mathrm{dl})$ & $29,4 \%$ & $10 \%$ & $\mathrm{NS}$ \\
Leucocitos/ $\mathrm{mm}^{3}$ & $6.626 \pm 2.218$ & $6.325 \pm 2.223$ & $\mathrm{NS}$ \\
Leucocitosis $\left(>11.000 / \mathrm{mm}^{3}\right)$ & $11,7 \%$ & $10 \%$ & $\mathrm{NS}$ \\
VSG (mm/12 hora) & $46,2 \pm 33,6$ & $24,7 \pm 21,7$ & NS \\
VSG elevada* & $52,9 \%$ & $20 \%$ & $\mathrm{NS}$ \\
\hline
\end{tabular}

VSG: velocidad de sedimentación globular. NS: no significación estadísti ca $(p \geq 0,05)$. *VSG $>40 \mathrm{~mm} / 1^{\mathrm{a}}$ hora.

\section{DISCUSIÓN}

En zonas como la nuestra donde la TBC y la brucelosis son endémicas, la mayor parte de los autores aceptan que la realización de pruebas diagnósticas invasivas (biopsia o punción-aspiración de las lesiones vertebrales o paravertebrales) ante la sospecha por el cuadro clínico-radiológico-serológico de ET o EB, debería limitarse a los casos en los que no hay respuesta al tratamiento empírico, o se sospeche otra entidad o una TBC multi-resistente $(2,7)$. Así en un tanto por ciento no despreciable de casos el diagnóstico de ET y EB es presuntivo, como en nuestra serie, en la que en el $48,1 \%$ de los casos no se dispuso de confirmación microbiológica. La exclusión de los pacientes sin un diagnóstico microbiológico definitivo 
supondría probablemente un sesgo en el intento de determinar posibles diferencias en las características de la ET y de la EB. Además, hay que destacar que el rendimiento diagnóstico de las pruebas invasivas no fue muy alto para la EB en nuestro estudio. La biopsia y/o punción-aspiración de las lesiones vertebrales o paravertebrales es necesaria con fines diagnósticos con mayor frecuencia en la ET que en la EB $(2-4,6,7)$, como ocurrió con nuestra serie, debido a la indudable ayuda diagnóstica que supone la serología en la EB.

En la mayoría de las series y en la nuestra, tanto la ET como la EB ocurren fundamentalmente en varones mayores de 50 años (3-9). La inmunosupresión fue infrecuente en nuetros pacientes, y se presentó con mayor frecuencia en la ET que en la EB, aunque sin alcanzar la significación estadística. Esta tendencia sin alcanzar la significación estadística también ha sido descrita por Colmenero y cols. en una amplia serie de nuestro medio (4). El tiempo de evolución de la clínica hasta el momento del diagnóstico fue significativamente mayor en nuestro grupo de pacientes con ET que en el grupo de EB, lo que coincide con lo descrito en otros estudios $(5,8,11)$, y puede explicarse al menos en parte por la mayor expresividad clínica de la EB. En nuestra serie encontramos una mayor frecuencia de fiebre/febrícula, dolor vertebral y pérdida de peso en la EB, de manera similar a otras series $(4,5,7)$, aunque en nuestro estudio no se alcanzó la significación estadística. No hubo diferencia significativa entre ambos grupos en la frecuencia de dolor vertebral, aunque debe destacarse que 2 casos del total de 17 de ET no presentaron dolor vertebral.

La localización lumbar seguida de la dorsal fue la más frecuente en nuestro estudio tanto en la ET como en la EB, siendo las localizaciones cervical y sacra raras. La localización más frecuente de la ET varía ampliamente. En algunas series la localización dorsal es la más frecuente, constituyendo aproximadamente el $55-75 \%$ del total $(4,7,9)$, mientras que en otros estudios como en el nuestro la localización más frecuente es la lumbar $(3,5)$. En el caso de la EB, la localización lumbar es la más frecuente en la mayoría de los estudios (4-10), resultado similar al nuestro. La frecuencia de la existencia de uno o varios abscesos paravertebrales y/o epidurales en la ET y en la EB también varía ampliamente de unas series a otras. Se ha descrito en el 20-78\% de los casos de ET y en el 5-76\% de los casos de EB $(4-7,10)$. Nosotros encontramos una mayor frecuencia de uno o varios abscesos paravertebrales y/o epidu- rales en la ET que en la EB, sin alcanzar la significación estadística, observación ya descrita en otras series $(4,5,10)$. Todos nuestros casos de ET y EB tuvieron evidencia de discitis en las pruebas de imagen conforme con la descripción clásica de las espondilitis infecciosas. No obstante, en una serie francesa de los últimas 2 décadas, la mitad de los casos de ET no presentaron evidencia de discitis, si bien dos tercios del total de los casos correspondió a inmigrantes, lo que puede se reflejo de un comportamiento distinto de la ET en otros grupos étni$\cos (3)$. La afectación de más de 2 cuerpos vertebrales fue rara en nuestro estudio, y ocurrió con una frecuencia similar en la ET y en la EB, contrariamente a lo descrito en otras series en las que la afectación de más 2 vértebras fue más frecuente en la EB $(4,10)$. La compresión medular fue más frecuente en la ET que en la EB, resultado similar al de otras estudios $(4,10)$, pero en nuestro caso nuevamente no se alcanzó la significación estadística. Las secuelas tras completar tratamiento fueron más frecuentes en la EB, sin alcanzar significación estadística, fundamentalmente por la ocurrencia de dolor vertebral residual, ya que la frecuencia de déficit motor residual fue similar en la ET y en la EB.

En el análisis de las características analíticas, encontramos una mayor frecuencia de anemia en la ET que en la EB, sin alcanzar la significación estadística. La leucocitosis fue infrecuente tanto en la ET como en la EB, ya que sólo estuvo presente en aproximadamente el 10\% de los casos. La velocidad de sedimentación globular fue más alta y estuvo elevada con mayor frecuencia en la ET, diferencias que estuvieron cerca de alcanzar la significación estadística $(\mathrm{p}=0,06)$.

En conclusión, la ET se caracterizó en nuestra serie por un mayor tiempo de evolución de la clínica hasta el momento del diagnóstico que la EB. También encontramos otras diferencias en el análisis de las características clínicas y analíticas, pero sin alcanzar la significación estadística. Debe tenerse en cuenta que nuestra serie de casos de ET y EB no es grande, y posiblemente no sea lo suficientemente amplia como para que se alcance la significación estadística en algunas de estas posibles diferencias entre los grupos de ET y EB. Según nuestros resultados, parecen exisitir algunas diferencias en las características clínicas y analíticas de la ET y de la EB que pueden ser de cierta ayuda en el diagnóstico diferencial de ambas entidades, y orientar el tratamiento empírico en los casos sin un diagnóstico definitivo microbiológico o a la espera de la confirmación diagnóstica.

\section{Bibliografía}

1. Anibarro García L, Vázquez-Gallardo R, Toubes Navarro ME, Penas Truque A, Lema Mougán R, Túñez Bastida V et al. Epidemiología de la tuberculosis en Galicia. An Med Interna (Madrid) 1999; 16: 290-296.

2. Watts HG, Lifeso RM. Tuberculosis of bones and joints. J Bone Joint Surg 1996; 78A: 288-298.

3. Pertuiset E, Beaudreuil J, Liote F, Horusitzky A, Kemiche F, Richette P et al. Spinal tuberculosis in adults. A study of 103 cases in a developed country, 1980-1994. Medicine (Baltimore) 1999; 78: 309-320.

4. Colmenero JD, Jiménez-Mejías ME, Sánchez-Lora FJ, Reguera JM, Palomino-Nicas J, Martos F et al. Pyogenic, tuberculous, and brucellar vertebral osteomyelitis: a descriptive comparative study of 219 cases. Ann Rheum Dis 1997; 56: 709-715.

5. Belzunegui J, Del Val N, Intxausti JJ, De Dios JR, Queiro R, González $\mathrm{C}$ et al. Vertebral osteomyelitis in northern Spain. Report of 62 cases. Clin Exp Rheumatol 1999; 17: 447-452.

6. Ariza J, Gudiol F, Valverde J, Pallares R, Fernández-Viladrich P, Rufi $\mathrm{G}$ et al. Brucellar spondylitis: a detailed analysis based on current fin- dings. Rev Infect Dis 1985; 7: 656-664.

7. Colmenero JD, Reguera JM, Martos D, Sánchez-de-Mora D, Delgado $\mathrm{M}$, Causse $\mathrm{M}$ et al. Complications associated with Brucella melitensis infection: a study of 530 cases. Medicine 1996; 75: 195-211.

8. González-Gay MA, García-Porrúa C, Ibáñez D, García-País MJ. Osteoarticular complications of brucellosis in an Atlantic area of Spain. J Rheumatol 1999; 26: 141-145.

9. Sharif HS, Aideyan OA, Clark DC, Madkour MM, Aabed MY, Mattsson TA et al. Brucellar and tuberculous spondylitis: comparative imaging features. Radiology 1989; 171: 419-425.

10. Cordero M, Sánchez I. Brucellar and tuberculous spondylitis. A comparative study of their clinical features. J Bone Joint Surg 1991; 73B: 100103.

11. González-Gay MA, García-Porrúa C, Cereijo MJ, Rivas MJ, Ibáñez D, Mayo J. The clinical spectrum of osteoarticualr tuberculosis in nonhuman immunodeficiency virus patients in a defined area of Northwestern Spain (1988-1997). Clin Exp Rheumatol 1999; 17: 663-669. 